\title{
Dopamine agonists and antipsychotics
}

\section{Mark E Molitch}

Division of Endocrinology, Metabolism and Molecular Medicine, Northwestern University, Feinberg School of Medicine, Chicago, Illinois, USA
Correspondence should be addressed to M E Molitch

Email

molitch@northwestern.edu

\section{Abstract}

There can potentially be a number of clinical interactions that could adversely affect patient outcomes in a patient with a prolactinoma and psychiatric disease that might require antipsychotic and dopamine agonist treatment. Dopamine agonists stimulate the dopamine D2 receptor, resulting in a decrease in prolactin (PRL) levels and in prolactinoma size but action on dopamine receptors in the meso-limbic system may rarely cause psychosis and more commonly cause impulse control disorders. The psychiatric benefits of antipsychotic agents involve blocking the D2 and other dopamine receptors but this blockade often also causes hyperprolactinemia.

In patients with macroprolactinomas and psychosis, observation, estrogen/progestin replacement, and surgery can be considered in addition to dopamine agonists. In those who require dopamine agonists for PRL and tumor size control, the introduction of antipsychotics may blunt this effect, so that higher doses of the dopamine agonists may be needed. Alternatively, antipsychotics that have less of a blocking effect at the D2 receptor, such as aripiprazole, can be tried, if appropriate. For patients already on antipsychotic drugs who are found to have a macroprolactinoma for which dopamine agonists are required, dopamine agonists can be initiated at low dose and the dose escalated slowly. However, such patients require careful monitoring of psychiatric status to avoid the rare complication of exacerbation of the underlying psychosis. Again, if appropriate, use of antipsychotics that have less of a blocking effect at the D2 receptor may allow lower doses of dopamine agonists to be used in this situation.

In a recent issue, Allard et al. report retrospective data from a multicenter study describing the management of 18 patients with macroprolactinomas and psychoses, focusing on the use of antipsychotic agents and dopamine agonists (1). To understand why these data are important for clinical practice, it is useful to review the pharmacology of how these drugs work and their psychiatric adverse effects.

As endocrinologists, we are familiar with dopamine agonists acting on dopamine D2 receptors to inhibit prolactin (PRL) secretion and decrease tumor size in patients with prolactinomas. In general, these medications are highly efficacious and well tolerated. However, psychiatric side effects from dopamine agonists are not uncommon and are thought to be related to activation of D2 and D3 receptors in the mesolimbic cortex (2). True psychosis, manifesting as hallucinations, mania or depression, is rare (2). However, impulse control disorders (ICD), the most common being hypersexuality (especially in men) and gambling, have been reported in up to $25 \%$ of patients taking dopamine agonists, compared to $8 \%$ in the general population (2). ICD have been reported more commonly in patients with Parkinson's disease than with prolactinomas, largely related to the higher doses used in the former. I have seen several patients with prolactinomas who experienced hypersexuality with both bromocriptine and cabergoline, although noted only in retrospect in most of these. In patients with dopamine agonist-related psychosis, the drugs must be stopped. In those with ICD, the symptoms improve with dose lowering and cease if the drug is stopped. It is important to actually ask patients and their family members specifically about these psychiatric adverse effects, as the abnormal behaviors may not be realized as being due to the drugs and often are hidden, even from family members.

The antipsychotic effects of the antipsychotic drugs are also mediated by dopamine D2 receptor blockade as well as D1, D3 and D4 receptor blockade in the

Published by Bioscientifica Ltd. 
mesolimbic area of the brain (3). Their extrapyramidal side effects are mediated through $\mathrm{D}_{2}$ receptors in the nigrostriatal area (3). The hyperprolactinemia from these drugs is mediated by blocking $\mathrm{D}_{2}$ receptors in the hypothalamic tuberoinfundibular system and on pituitary lactotrophs (4). Not all antipsychotic drugs increase PRL levels. The first-generation agents - the phenothiazines, thioxanthenes and butyrophenones - all raised PRL levels. Of the later second-generation agents, often referred to as 'atypical' antipsychotics, risperidone and its derivatives and molindone are the most common causes of hyperprolactinemia (4). Clozapine, olanzapine, quetiapine, ziprasidone, and aripiprazole much less commonly cause hyperprolactinemia, likely because they only transiently and weakly bind to tuberoinfundibular D2 receptors and to their having agonist as well as antagonist activity at $\mathrm{D} 2$ receptors (4).

Based on the information discussed above, it can be anticipated that there could potentially be a number of interactions that could adversely affect patient outcomes in a patient with a prolactinoma and psychiatric disease that might require antipsychotic and dopamine agonist treatment. A patient whose prolactinoma size and PRL levels are controlled by a dopamine agonist could have a worsening of such control if a dopamine receptor blocker is introduced. Conversely, if a patient with schizophrenia or other psychiatric condition requiring an antipsychotic medication is also found to have a prolactinoma, introduction of a dopamine agonist could potentially cause an exacerbation of the psychiatric condition.

These potential interactions were studied retrospectively in this report by Allard et al. in 18 patients with macroprolactinomas and psychiatric disease seen in several hospitals in France and Belgium (1). With respect to the effect of the antipsychotic agent on the efficacy of the dopamine agonist on prolactinoma control, they did not have data from patients in whom the dopamine agonist was being used alone with subsequent addition of the antipsychotic agent. However, when they compared the results in these patients to others they have treated who were not taking antipsychotic agents, the ability to normalize PRL levels and to achieve significant tumor shrinkage seemed to be reduced. But this comparison was not performed in a statistically rigorous manner. On the other hand, they found that in six patients, switching antipsychotic agents to other agents less prone to cause hyperprolactinemia did not alter the efficacy of the dopamine agonists. Other case reports have demonstrated the ability of antipsychotic agents to decrease the efficacy of dopamine agonists (5).
Allard et al. also evaluated the possibility of a psychiatric exacerbation by the dopamine agonists (1). Of their 18 patients, nine experienced psychosis relapses but only five of these nine were taking dopamine agonists at the time and three of these five were not taking antipsychotic agents at the time of the relapse. Because of these issues, the authors were unable to draw any firm conclusions regarding dopamine agonist exacerbation of psychoses. There are case reports in the literature showing both exacerbation (6) and lack of exacerbation (7) of psychotic symptoms with dopamine agonists.

What conclusions can we draw from this study and others? Certainly the two drug classes can interact but the degree of this interaction is variable, dose dependent, and dependent upon which antipsychotic agent is used. Not all patients with prolactinomas need to be treated with dopamine agonists. Postmenopausal women often need no treatment and can just be observed with periodic monitoring of PRL levels. Younger women not interested in fertility can often be treated with estrogen/progestin replacement, again with monitoring of PRL levels. Transsphenoidal surgery can also be an option if the tumor size/extension indicate a high likelihood of cure in expert surgical hands. If monitoring of PRL levels in women who are not treated or treated with estrogen/progestin reveals a rising PRL level, repeat magnetic resonance imaging (MRI) may be indicated and dopamine agonist or surgery done if tumor size is increasing. For patients with macroadenomas that require treatment with dopamine agonists, the issues are more complex. The concomitant use of an antipsychotic may partially block the action of the dopamine agonist, so that higher doses may be needed. Caution is needed if the cabergoline dose exceeds $2 \mathrm{mg} /$ week regarding monitoring for cardiac valvular abnormalities (8). If the psychiatric consultant agrees, then a trial of switching to an antipsychotic agent less likely to cause hyperprolactinemia, such as aripiprazole, could be also be tried.

The other scenario is the patient with a psychiatric condition being treated with an antipsychotic agent who develops symptoms related to hyperprolactinemia, such as galactorrhea and amenorrhea, or to mass effects, such as headaches, and is then found to have a prolactinoma on investigation with MRI. The same analysis as discussed above is needed regarding treatment, i.e. no treatment, estrogen/progestin replacement, surgery, or dopamine agonist. If the patient has a macroadenoma requiring dopamine agonist for control of tumor size, the same considerations pertain as well. Although dopamine agonists can cause an exacerbation of the psychosis in such 
patients, it appears that this is quite rare. Nonetheless, the dopamine agonist should be initiated with caution only after careful discussion with the patient, the patient's family and the consulting psychiatrist. The drugs should be started at a very low dose, for example, cabergoline at $0.25 \mathrm{mg}$ once a week, with dose escalation done slowly with careful monitoring for worsening of psychiatric symptoms. The lowest dose that will control the PRL levels and tumor size should be used. Because of the blocking effect of the antipsychotic agent, switching to a drug like aripiprazole can be useful, if the psychiatric consultant agrees, in order to avoid high doses of the dopamine agonist.

Allard et al. (1) have highlighted important issues in the management of patients with prolactinomas and psychiatric disease. The analyses discussed above of the use of these drugs in the individual patient will generally permit a successful outcome.

\section{Declaration of interest}

The author- declares that there is no conflict of interest that could be perceived as prejudicing the impartiality of this commentary.

\section{Funding}

The author has received research grant funding from Novartis, Chiasma, Janssen, Strongbridge, and Crinetics and has received consultation honoraria from Janssen and Chiasma.

\section{References}

1 Allard L, Albarel F, Bertherat J, Caron P, Cortet C, Courtillot C, Delemer B, Jublanc C, Maiter D, Nunes M-L et al. Efficacy and safety of dopamine agonists in patients treated with antipsychotics and presenting a macroprolactinoma. European Journal of Endocrinology 183 221-231. (https://doi.org/10.1530/EJE-20-0125)

2 Ioachimescu AG, Fleseriu M, Hoffman AR, Vaughan TB III \& Katznelson L. Psychological effects of dopamine agonist treatment in patients with hyperprolactinemia and prolactin-secreting adenomas. European Journal of Endocrinology 2019180 31-40. (https://doi. org/10.1530/EJE-18-0682)

3 Miyamoto S, Duncan GE, Marx CE \& Lieberman JA. Treatments for schizophrenia: a critical review of pharmacology and mechanisms of action of antipsychotic drugs. Molecular Psychiatry 200510 79-104. (https://doi.org/10.1038/sj.mp.4001556)

4 Molitch ME. Drugs and prolactin. Pituitary 200811 209-218. (https://doi.org/10.1007/s11102-008-0106-6)

5 Robbins RJ, Kern PA \& Thompson TL II. Interactions between thioridazine and bromocriptine in a patient with a prolactinsecreting pituitary adenoma. American Journal of Medicine 198476 921-923. (https://doi.org/10.1016/0002-9343(84)91010-6)

6 Chang S-C, Chen C-H, Lu M-L. Cabergoline-induced psychotic exacerbation in schizophrenic patients. General Hospital Psychiatry 200830 378-380. (https://doi.org/10.1016/j. genhosppsych.2007.11.002)

7 Tollin SR. Use of the dopamine agonists bromocriptine and cabergoline in the management of risperidone-induced hyperprolactinemia in patients with psychotic disorders. Journal of Endocrinological Investigation 200023 765-770. (https://doi. org/10.1007/BF03345068)

8 Stiles CE, Tetteh-Wayoe ET, Bestwick JP, Steeds RP \& Drake WM. A meta-analysis of the prevalence of cardiac valvulopathy in patients with hyperprolactinemia treated with cabergoline. Journal of Clinical Endocrinology \& Metabolism 2019104 523-538. (https://doi. org/10.1210/jc.2018-01071)

Received 1 June 2020

Accepted 3 June 2020 